\title{
Assessment of Information Redundancy in ECG Signals
}

\author{
B Acar, L Özçakir, H Köymen \\ Bilkent University, Department of Electrical and Electronics Engineering, Ankara, Turkey
}

\begin{abstract}
In this paper, the morphological information redundancy in standard 12 lead ECG channels is studied. Study is based on decomposing the ECG channels into orthogonal channels by an SVD based algorithm and then reconstructing them. Then 7 of 8 independently recorded ECG channels are decomposed and the missing channel is reconstructed from these orthogonal channels. Thus the unique morphological information content of each ECG channel is assessed through the loss of clinical information in the reconstructed signal. A comparison of the clinical parameters measured from the reconstructed and original ECG is reported.
\end{abstract}

\section{Introduction}

Several modeling techniques were reported in the literature that models the ECG by fitting Gaussian curves [1], by using wavelets [2] and by calculating the Fourier Series coefficients [3]. Tai [4] used a Markov system to enhance subband coding of ECG where he considered intersample redundancy in estimating next coming sample values from the previous ones. In these approaches redundancy in ECG is evaluated from a coding point of view. Lux et.al. [5] emphasizes the importance of using a small number of optimally selected lead in ECG interpretation and hence addresses information redundancy in standard 12 lead ECG channels.

We decomposed these signals into orthogonal components by an online SVD based algorithm [6], for noise elimination in exercise ECG. The decomposed signals are reconstructed and compared with the original signal in terms of measured morphological parameters, $\mathrm{P}$-wave, $\mathrm{R}$-wave, $\mathrm{T}$-wave amplitudes, $\mathrm{R}$-wave duration and $\mathrm{j}$-point elevation.

\section{Method}

50 exercise ECG records of lengths between 6:20 to 20:00 minutes are analyzed. Data was sampled at 500 samples/second and at 12 bits resolution. All these data are recorded during stress tests performed under Bruce protocol.

The first 30 seconds of each record is used for training the SVD algorithm and the rest is used for the analysis. All data is divided into 20 second periods, which are called a segment. Thus we have 30 segments for a 10 minute data. Two different reconstructed data sets are produced: (i) $8 \mathrm{ECG}$ channels are decomposed by a SVD based algorithm and reconstructed using only 3 orthogonal channels which contain the ECG information [6]. This set will be referred to as $8 \mathrm{D}$. (ii) 7 out of 8 channels are decomposed into its orthogonal components and then the missing channel is reconstructed by the reconstruction coefficients of the previous segment obtained for $8 \mathrm{D}$ analysis. The decomposition is done with $8 \mathrm{ECG}$ channels until the end of the previous segment and channel exclusion is done only during the current segment. This process is repeated for each segment of each record. This set will be referred to as $7 \mathrm{D}$. The segments with high noise, in which the SVD algorithm excluded the noisy channel automatically, are also excluded from the analysis. An average beat is calculated for each of the rest of the segments. Clinical parameter measurements are made on these average beats for each segment included in the analysis.

In this paper, the comparison of R-wave, P-wave, T-wave amplitudes, R-wave duration and $\mathrm{j}$-point elevation measurements made on three sets of data are presented. The comparison of wave amplitudes and duration is made for precordial lead V5 and limb lead DIT. These measurements on average beats for cach scgment is performed using the IBM-Mt.Sinai hospital program. $J$-point clevation comparison is presented for all channels, but on the other hand for a single segment.

\section{Discussion}

$\mathrm{J}$-point elevation is measured on the average beats of the first segment of each record for all channels. Figure 1 shows measurements done on input, 8D and 7D signals. Both of the measurements from $8 \mathrm{D}$ and $7 \mathrm{D}$ signals approximate the measurements from input sig- 

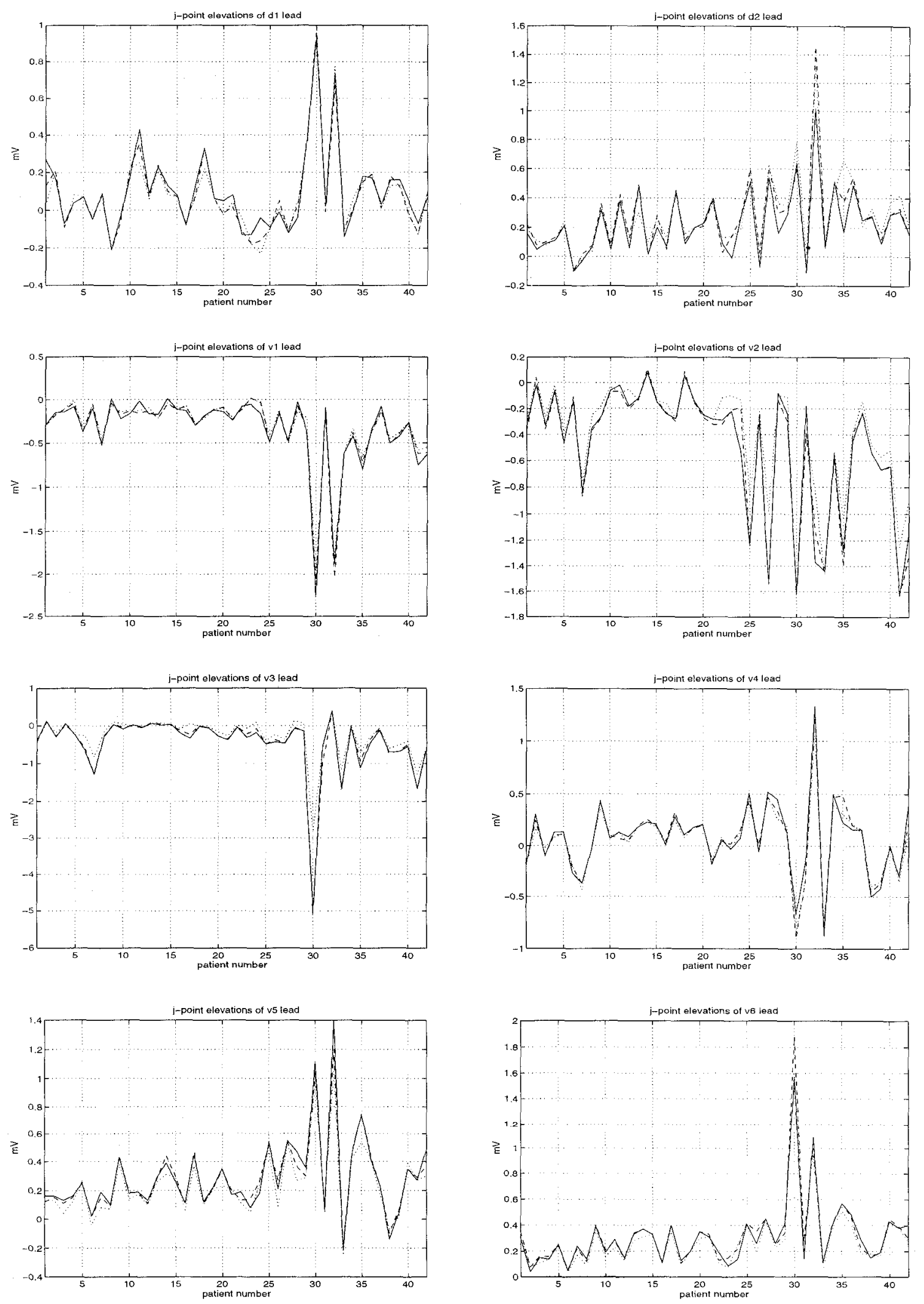

Figure 1: J-point Elevation (-:Input, , -:8D , ..:7D) 

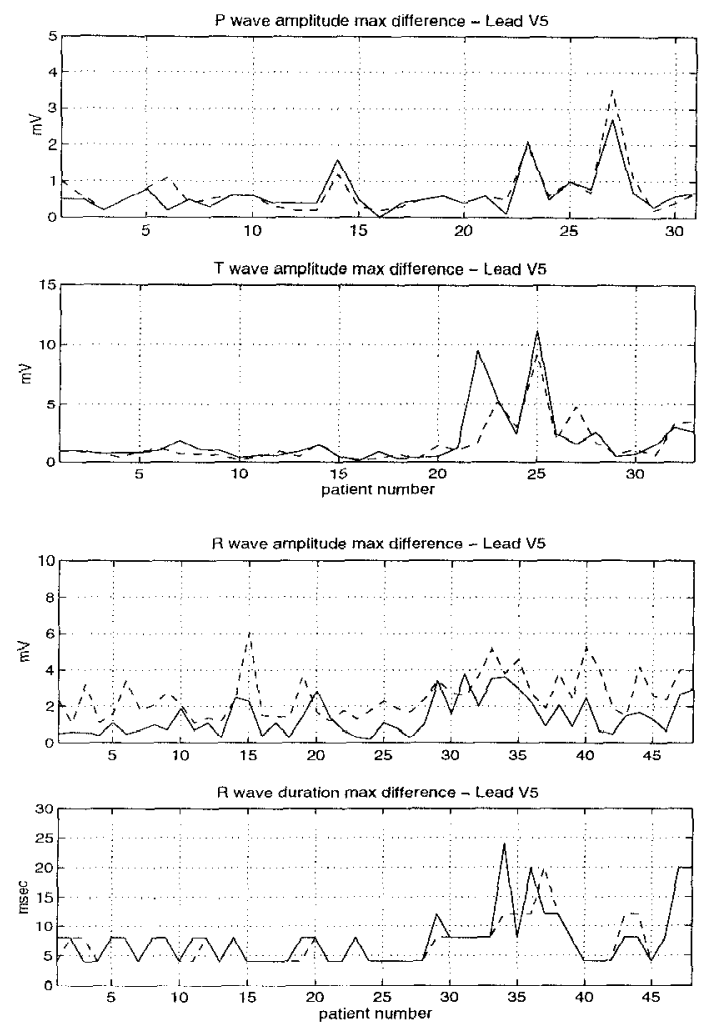
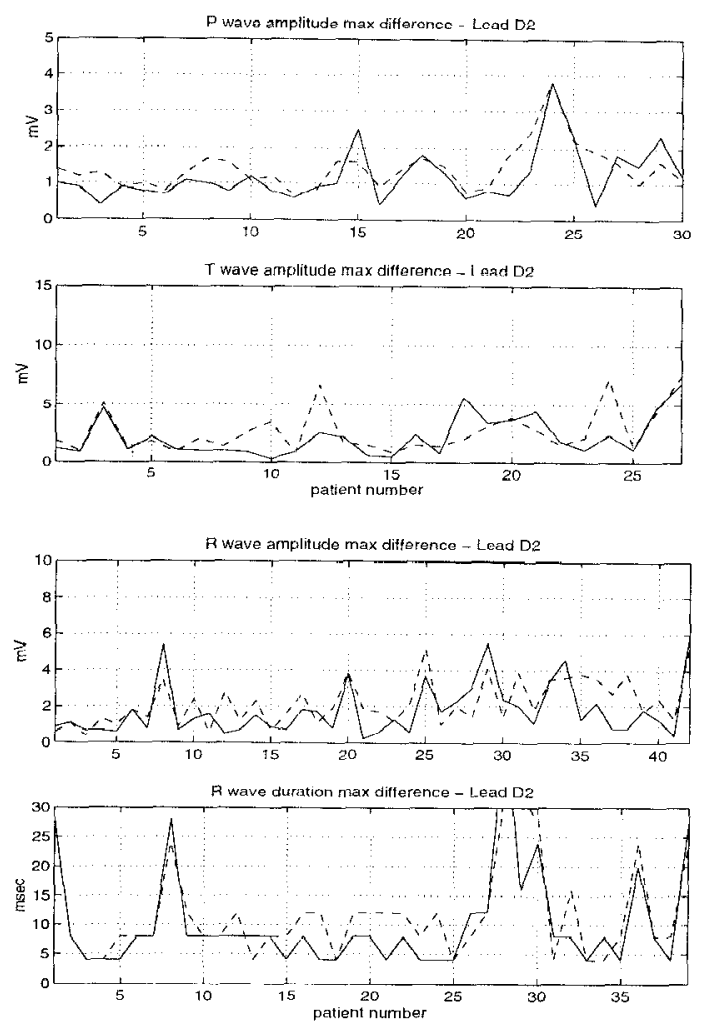

Figure 2: Max. absolute difference in measured clinical parameters (-:Inp-8D , - -:Inp-7D)

nals well. Since the data is recorded during stress tests, the input signals are also noisy. Reconstructed signals, however, are less noisy due to noise elimination during reconstruction [6]. It can be conjectured that the relative difference between the input channel and reconstructed channels are partly caused by this fact. Table 1 shows the mean absolute difference between the input, $8 \mathrm{D}$ and $7 \mathrm{D}$ signals. The difference in $7 \mathrm{D}$ signals is somewhat higher than the difference in $8 \mathrm{D}$ signals. As can be observed in Figure 1, j-point level is very well approximated in reconstructed signals for this patient data set.

The IBM-Mt.Sinai Hospital program is developed to analyze rest ECG. The measurement part of the program occasionally fails to detect the complexes and hence we encountered measurement problems when the linked medians composed of median beats of every segment is processed by the program. We had to exclude the records with such detection errors. As a result, we used different sets of data in the analysis of different parameters. The records with noise and artifacts are maintained in the data set. The analysis program made some measurement errors in such records.

Figure 2 shows the maximum absolute differences in D2 and V5 among all segments for each record an-

\begin{tabular}{|c|c|c|}
\hline Lead & Input-8D (mV) & Input-7D $(\mathrm{mV})$ \\
\hline DI & 0.03 & 0.04 \\
\hline DII & 0.04 & 0.07 \\
\hline V1 & 0.04 & 0.06 \\
\hline V2 & 0.04 & 0.11 \\
\hline V3 & 0.05 & 0.18 \\
\hline V4 & 0.05 & 0.06 \\
\hline V5 & 0.03 & 0.06 \\
\hline V6 & 0.03 & 0.04 \\
\hline
\end{tabular}

Table 1: Mean absolute difference in j-point clevation

alyzed. The large differences are mainly due to measurement errors, such as patient 27 in V5 P-wave amplitude. The average beats of this patient are given in Figure 3 - Record 1, where a very good reconstruction is observed. The average beats obtained from input, $8 \mathrm{D}$ and $7 \mathrm{D}$ signals, in two typical records, are shown. 8D signals are almost the same as input signals. However, measurements from 7D signals are somewhat more erroneous. It is observed that the highest differences occur at P-wave and T-wave. DII, in 7D signal, has much higher deviation from the input signal when compared 

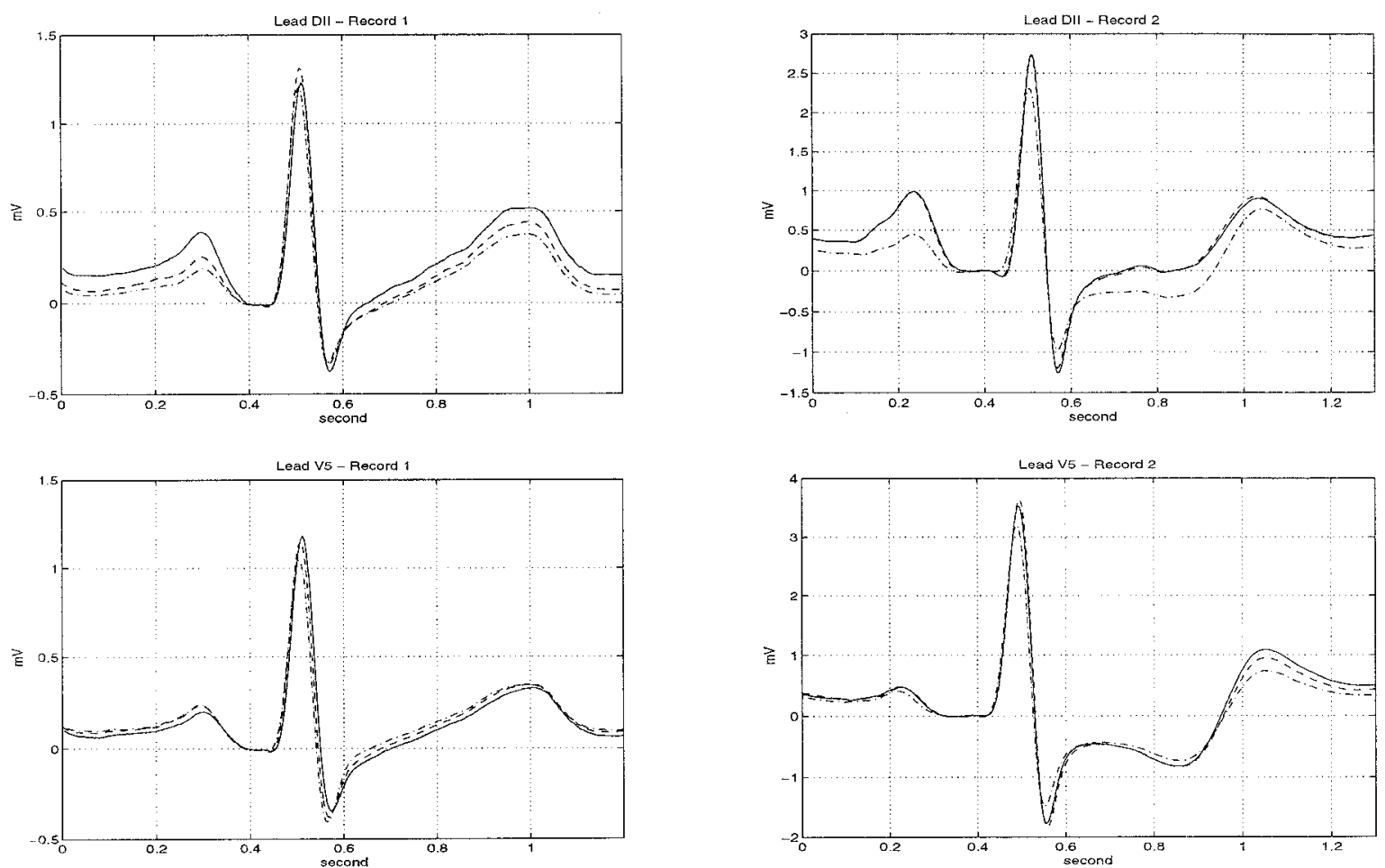

Figure 3:-:Input , - -:8D , -.:7D

with other derivations. The relatively large differences in R-wave amplitude, observed in Figure 2, is due to the large values of that parameter.

\section{Conclusion}

In this study, we first showed that the morphological information is preserved during the decomposition and reconstruction process given in [6]. This shows that the relevant information in ECG can be represented in a 3 dimensional space without significant loss of information.

Then we showed the information redundancy in standard 12 lead ECG channels. Each one of the 8 ECG chamnels is reconstructed from the information present in other 7 channels and it is observed that the clinical information can be regained when compared to the original channel.

Due to the detection and measurement problems mentioned above, further assessment of these and other clinical parameters must be done with an improved detection and measurement tool suitable for exercise ECG data.

\section{References}

[1] Suppappola S., Sun Y., Chiaramida S.A., "Gaussian Pulse Decomposition - An Intuitive Model of Electrocardiogram Wave-Forms," Annals Of Biomedical Engineering, vol. 25, Iss 2,pp. 252-260, 1997.

[2] Hilton M.L., "Wavelet and Wavelet Packet Compression of Electrocardiograms," IEEE Trans. On Biomedical Eng., vol. 44, Iss 5,pp. 394-402, 1997.

[3] Alnashash H.A.M., "A Dynamic Fourier Series for the Compression of ECG Using FFT and Adaptive Coefficient Estimation," Medical Engineering \&\& Physics, vol. 17, Iss 3,pp. 197-203, 1995.

[4] Tai S.C., "Improving the Performance of Electrocardiogram Subband Coder by Extensive Markor System," Medical \& Biological Engineering 8 Computing, vol. 33, Iss 3,pp. 471-475, 1995.

[5] Lux R.L., Macleod R.S., Fuller M., Green L.S., Kornreich F., "Estimating ECG Distributions from Small Numbers of Leads," Journal of Electrocardiology, vol. 28 , Iss S,pp. 92-98, 1995.

[6] Acar B., Köymen H., "Online Exercise ECG Signal Orthogonalization" Proceedings of Computers In Cardiology'96, pp. 237-240, 1996.

Address for correspondence:

Bilkent University

Eng. Fac. EE Dept.

06533 Ankara. Turkey

tel/fax: $++90-312-2664307$

e-mail: buraka@ee.bilkent.edu.tr 